\title{
IGF2BP3 as a potential tissue marker for the diagnosis of esophageal high-grade intraepithelial neoplasia
}

\author{
Jingjing Zhang ${ }^{1, *}$ \\ Qing Ji ${ }^{2}, *$ \\ Chunhua Jiao ${ }^{3, *}$ \\ Lihua Ren ${ }^{4}$ \\ Ye Zhao ${ }^{4}$ \\ Yanfang Chen ${ }^{4}$ \\ Ruihua $\mathrm{Shi}^{4}$ \\ Yadong Feng ${ }^{4}$
}

'State Key Laboratory of Reproductive Medicine, Department of Prenatal Diagnosis, Obstetrics and Gynecology Hospital Affiliated to Nanjing Medical University, Nanjing, ${ }^{2}$ Department of Emergency, Jingjiang People's Hospital, Jingjiang, ${ }^{3}$ Department of Gastroenterology, First Affiliated Hospital with Nanjing Medical University, ${ }^{4}$ Department of Gastroenterology, Zhongda Hospital, School of Medicine, Southeast University, Nanjing, People's Republic of China

*These authors contributed equally to this work
This article was published in the following Dove Press journal:

OncoTargets and Therapy

I August 2017

Number of times this article has been viewed

Background: The clinical significance of insulin-like growth factor-II mRNA-binding protein-3 (IGF2BP3) in esophageal high-grade intraepithelial neoplasia (HGIN) is not clear. This study was designed to characterize the expression of IGF2BP3 in HGIN.

Patients and methods: IGF2BP3 expression was evaluated by Western blot analyses in 12 cases and by immunohistochemistry (IHC) in 112 cases. The associations between IGF2BP3 expression in HGIN and the clinicopathological parameters were examined.

Results: Moderate to strong IGF2BP3 expression was present in HGIN samples. Using IHC, it was found that IGF2BP3 was positive in $68(60.71 \%)$ cases. Intense IHC of IGF2BP3 in HGIN was associated with a deeper lesion depth, and the lesion depth was the only predictor of the positive expression of IGF2BP3.

Conclusion: Our results suggested that IGF2BP3 may be a supplementary tissue marker for preoperative diagnosis of HGIN.

Keywords: esophageal squamous cell carcinoma, precancerous lesion, immunohistochemistry detection, early diagnosis

\section{Introduction}

Esophageal squamous cell carcinoma (ESCC) is the predominant histological form of esophageal cancer and is one of the most lethal digestive tract malignancies in eastern Asia. ${ }^{1}$ Most cases of ESCC are diagnosed at an advanced stage and are with a poor prognosis. ${ }^{1}$ However, ESCC diagnosed at an early stage has a satisfactory 5 -year survival after resection, ${ }^{1}$ so the early detection of ESCC is important. Currently available biomarkers, such as serum squamous cell carcinoma antigen, carcinoembryonic antigen, and cytokeratin-19 fragment, have limited use in the early detection of ESCC because of a lack of sufficiently high diagnostic sensitivity and specificity. ${ }^{2}$

During the formation of ESCC, an atypical proliferation is observed, initially confined to the lower part of the squamous epithelium, then extending to the entire epithelium and the lamina propria. ${ }^{3}$ High-grade intraepithelial neoplasia (HGIN) is characterized by noninvasive neoplastic epithelial proliferation ${ }^{4}$ during the tumorigenesis of ESCC. HGIN is a precursor lesion of ESCC, and an HGIN $>15 \mathrm{~mm}^{5}$ is one of the indications for endoscopic submucosal dissection (ESD) and is indicative of a high risk of developing invasive ESCC. ${ }^{6}$ The diagnosis of HGIN depends on histological evaluations; however, small tissue specimens from biopsies often present a challenge for pathologists. A reliable tissue marker for the diagnosis of HGIN will therefore assist in the histological diagnoses. 
The insulin-like growth factor-II mRNA-binding protein-3 (IGF2BP3), also known as IMP3, was first reported in pancreatic cancer and was identified as a novel K homology domain-containing protein. ${ }^{7}$ In human beings, IGF2BP3 is expressed in the developing epithelium, muscle, and placenta during embryogenesis, while low levels of IGF2BP3 are detected in normal adult tissues. ${ }^{8}$ IGF2BP3 overexpression is detected in some cancers, including pancreatic, esophageal, lung, and gastric cancers. ${ }^{7,9-11}$ IGF2BP3 is also involved in the regulation of cell proliferation by targeting IGF-2. ${ }^{12}$ The overexpression of IGF2BP3 has been detected in ESCC and esophageal adenocarcinoma and its precancerous lesion. ${ }^{9}{ }^{9}$ However, the status of IGF2BP3 in HGIN is unknown. The aim of this study was therefore to investigate the expression of IGF2BP3 in HGIN.

\section{Patients and methods Ethics and patients}

This study was approved by the ethics committees of the First Affiliated Hospital of Nanjing Medical University and Zhongda Hospital. Patients newly diagnosed with HGIN $>15 \mathrm{~mm}$ and who received ESD at the First Affiliated Hospital with Nanjing Medical University and Zhongda Hospital between January 2011 and June 2014 were enrolled. Written informed consent was obtained from each patient. Final pathological diagnoses of all specimens, including neoplastic lesions confined to the squamous epithelium, HGIN, the pathological T (pT) stage, and the R0 resection without lateral or basal residence, were confirmed by two pathologists. In total, 112 patients ( 74 cases from the First Affiliated Hospital with Nanjing Medical University and 38 cases from Zhongda Hospital) were included, with 44 females and 68 males, aged 37-82 years. There were 21 cases of in situ carcinoma (pT1a-EP [M1]), 38 cases of tumor invasion of the lamina propria mucosa (pT1a-LPM [M2]), and 53 cases of tumor invasion of the entire mucosa layer (pT1a-MM [M3]). The clinicopathological features are listed in Table 1.

\section{Western blotting}

Specimens after ESD were subjected to Lugol's iodine staining, ${ }^{14}$ and HGIN tissues were taken from the nonstaining areas with a biopsy forceps. Total protein was extracted from the HGIN tissues. Approximately $40 \mu \mathrm{g}$ of protein was separated by $10 \%-15 \%$ sodium dodecyl sulfate polyacrylamide gel electrophoresis and transferred to polyvinylidene difluoride membranes (Pierce ${ }^{\mathrm{TM}}$; Thermo Fisher Scientific, Waltham, MA, USA). The membranes were incubated with a monoclonal rabbit antibody against IGF2BP3 (ab109521;
Table I The clinicopathological features of enrolled patients

\begin{tabular}{ll}
\hline Characteristics & $\mathbf{N}=\mathbf{I ~ I ~} \mathbf{2}$ \\
\hline Gender & 68 \\
Male & 44 \\
Female & $37-82 ; 61.28 \pm 10.76$ \\
Age (years), range; mean \pm SD & \\
Location & 32 \\
Upper & 31 \\
Middle & 49 \\
Lower & \\
Length & 82 \\
$<5$ cm & 30 \\
$>5$ cm & \\
Circumferential extent & 30 \\
$<1 / 2$ & 46 \\
I/2-2/3 & 36 \\
$>2 / 3$ & \\
PT stage & 21 \\
PTla-EP & 38 \\
PTla-LPM & 53 \\
PTla-MM & \\
\hline
\end{tabular}

Notes: pT stage: pathological T stage; pT la-EP: MI, carcinoma in situ; pT la-LPM: $M 2$, tumor-invaded lamina propria mucosa; $\mathrm{PT}$ Ia-MM: M3, tumor invaded the whole layer of mucosa.

Abcam, Cambridge, MA, USA). After washing with a mixture of tris-buffered saline and Tween 20 the blots were incubated with horseradish peroxidase-conjugated secondary antibody, and the bands were detected using an enhanced chemiluminescence detection system (Pierce, Thermo Fisher Scientific).

\section{Immunohistochemistry (IHC)}

IHC was performed on $5 \mu \mathrm{m}$ sections of formalin-fixed, paraffin-embedded tissue specimens from ESD. IHC was performed according to a standard protocol using a monoclonal rabbit anti-human IGF2BP3 antibody (ab109521; Abcam). The procedures of IHC targeting IGF2BP3 in this study were similar with those in previous studies. ${ }^{9}{ }^{13}$ All sections were deparaffinized in xylene, rehydrated through a graded ethanol series, and then incubated in $3 \% \mathrm{H}_{2} \mathrm{O}_{2}$ solution for $10 \mathrm{~min}$ at room temperature. Antigen retrieval was performed with $0.01 \mathrm{M}$ citrate buffer (pH 6.0) at $100^{\circ} \mathrm{C}$ for $20 \mathrm{~min}$. Endogenous peroxidase activity was blocked with $0.3 \% \mathrm{H}_{2} \mathrm{O}_{2}$ in methanol for $20 \mathrm{~min}$, and nonspecific binding was blocked with $10 \%$ normal serum for 20 min followed by a buffer wash. The sections were incubated with the primary antibody at a dilution of $1: 50$ overnight at $4^{\circ} \mathrm{C}$. The sections were then incubated with secondary goat anti-rabbit IgG (Santa Cruz Biotechnology Inc., Dallas, TX, USA) at a dilution of 1:200 at $37^{\circ} \mathrm{C}$ for $30 \mathrm{~min}$. Finally, the sections were treated with diaminobenzidine, counterstained with $0.1 \%$ hematoxylin, and coverslipped. Staining of IGF2BP3 was positive when at 
least $10 \%$ or more of the neoplastic cells in the section were immunoreactive with the anti-IGF2BP3 antibody. Intensity was scored as 0 (negative), 1+ (weak), 2+ (moderate), and $3+$ (strong). The status of IGF2BP3 expression was assessed by two pathologists.

\section{Statistical analysis}

All data were expressed as mean \pm standard deviation. SPSS statistical software for Windows, version 20.0 (SPSS Inc., Chicago, IL, USA), was used for analyses using the chi-squared test, one-way analysis of variance, and linear regression analyses. A value of $P<0.05$ was considered to be statistically significant.

\section{Results}

\section{Expression of IGF2BP3 in HGIN}

A total of 12 samples of HGIN tissues were used for the evaluation of IGF2BP3 expression by Western blotting. Seven samples showed moderate to strong expression, and five cases showed negative to weak expression (Figure 1). IHC staining of IGF2BP3 was performed to validate the results of Western blotting. In the 112 samples, $68(60.71 \%)$ cases showed positive expression of IGF2BP3, which was characterized by dark brown staining in the cytoplasm of the neoplastic cells (Figure 2). The positive staining was homogeneous in different areas of the neoplastic lesions. The remaining 44 cases showed negative expression of IGF2BP3. In noncancerous areas, the IGF2BP3 expression was negative. The staining intensity of IGF2BP3 was higher in pT1a-MM than that in pT1a-EP and pT1a-LPM (Table 2).

\section{Correlation between IGF2BP3 expression and clinicopathological parameters}

The positive expression of IGF2BP3 in HGIN was associated with deeper lesion depth $(P=0.042$; Table 3$)$. No significant

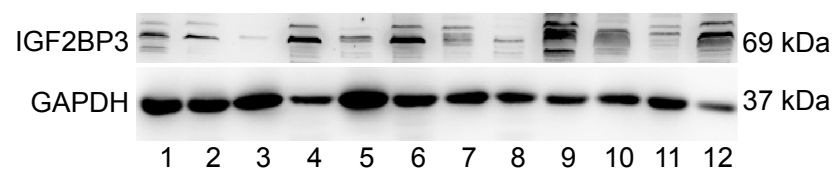

Figure I Analyses of IGF2BP3 expression in HGIN by Western blotting.

Notes: A total of 12 samples were analyzed. According to the final pathological diagnosis of 12 samples, the first, second, and fifth were MI; the third, sixth, seventh, and ninth were M2; and the remaining were M3. Seven cases showed moderate to strong IGF2BP3 expression, and five cases showed negative to weak expression. The relative expression of proteins was normalized to GAPDH.

Abbreviations: GAPDH, glyceraldehyde 3-phosphate dehydrogenase; HGIN, highgrade intraepithelial neoplasia; IGF2BP3, insulin-like growth factor-II mRNA-binding protein-3. difference of IGF2BP3 expression was observed in other parameters such as age, gender, location, and the extent and length of the neoplasm.

\section{Predictors of IGF2BP3 expression in HGIN}

The results of the univariate and multiple linear regression analyses of the correlation between the positive expression of IGF2BP3 and the predictors are provided in Table 4. The lesion depth was the only predictor of the positive expression of IGF2BP3 in HGIN. Other parameters, such as age, gender, location, and the extent and length of the neoplasm, were not correlated with the positive expression of IGF2BP3.

\section{Discussion}

The accurate preoperative diagnosis of HGIN is very important for patient management. ${ }^{5}$ The histological diagnosis of HGIN is usually straightforward when substantial neoplastic tissue is present in the resection or biopsy specimens. However, the diagnosis of HGIN may be challenging in routine biopsy because few neoplastic tissue is available. Such diagnosis in difficult cases is vital because HGIN may need esophageal resection and carry significant patient morbidity and mortality. ${ }^{5,6}$ New tissue markers may provide a supplement to histological diagnosis for HGIN in difficult cases.

The overexpression of IGF2BP3 has been reported in some tumors, ${ }^{9-13,15-19}$ including ESCC, and has been reported to be involved in cell proliferation, invasion, and the advanced disease stage. In squamous epithelial tumors, ${ }^{9,16-19}$ IGF2BP3 has been claimed as a potential marker for diagnosis. But, to the best of our knowledge, IGF2BP3 expression in HGIN has not been investigated. In this study, we evaluated the expression of IGF2BP3 in HGIN, which is the precursor lesion of ESCC. We showed the expression pattern of IGF2BP3 in HGIN. The negative expression of IGF2BP3 was present in noncancerous areas, and the positive expression was present only in neoplastic lesions. The expression pattern of IGF2BP3 in HGIN was consistent with it in previous studies. ${ }^{9-13,16-19}$ The positive expression of IGF2BP3 in HGIN, which was featured as cytoplasmic staining pattern, was similar with its expression in other squamous epithelial tumors, such as the esophagus, ${ }^{9}$ the skin, ${ }^{16}$ the head and neck, ${ }^{17}$ the tongue, ${ }^{18}$ and the uterine cervix. ${ }^{19}$ In pT1a-EP and pT1a-LPM, the positive expression of IGF2BP3 was predominantly located at the basal layer of the epithelium. In pT1a-MM, the positive expression of IGF2BP3 was characterized by immunoreactive staining in the whole epithelial layer of neoplastic lesions. This is consistent with the tumorigenesis of ESCC 

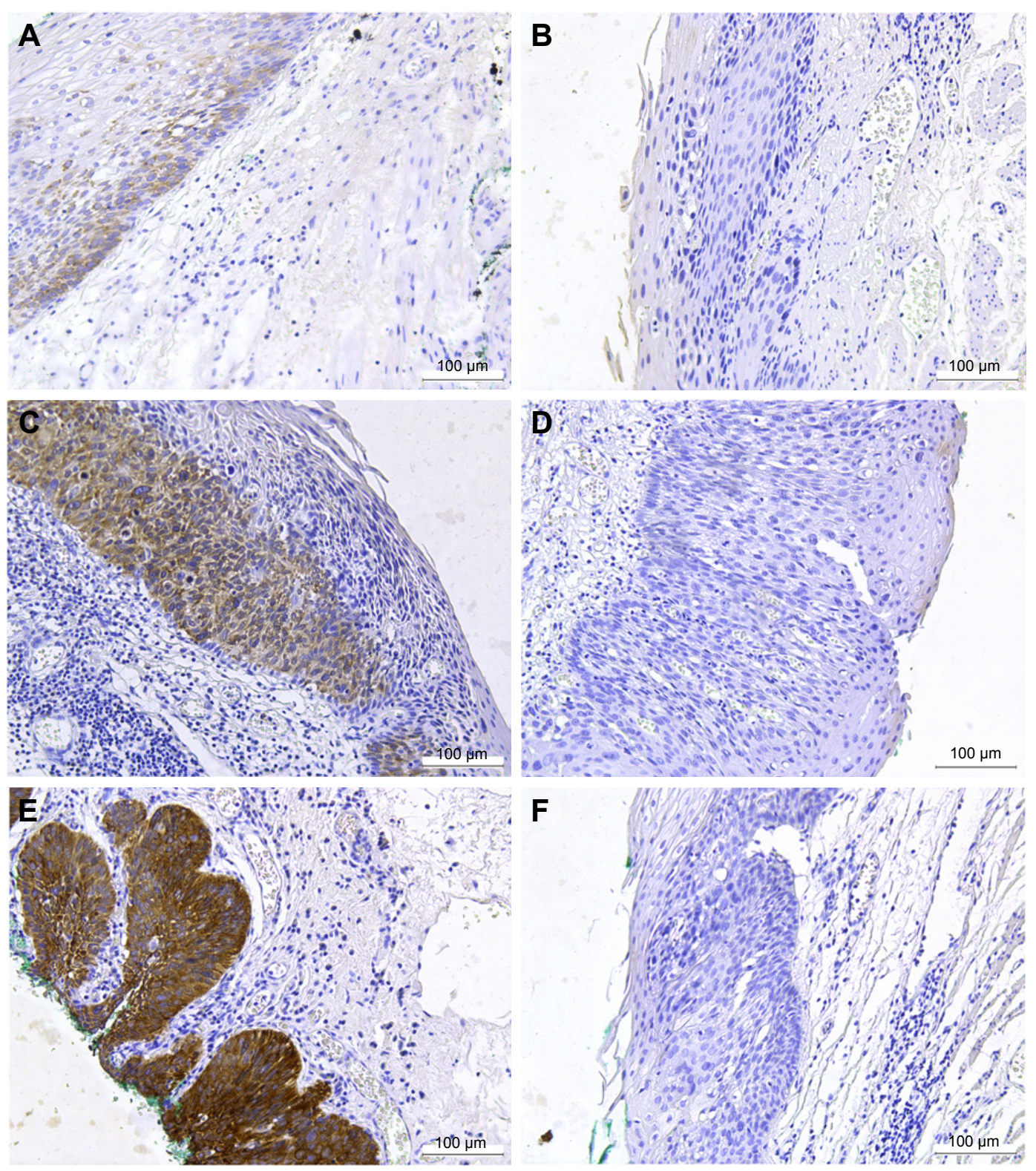

Figure 2 Analysis of IGF2BP3 expression in HGIN by IHC.

Notes: All images were captured at 200× magnification. Positive (A) and negative (B) staining of IGF2BP3 in PTIa-EP (MI). Positive (C) and negative (D) staining of IGF2BP3 in PTIa-LPM (M2). Positive (E) and negative (F) staining of IGF2BP3 in PTla-MM (M3). Positive staining of IGF2BP3 was characterized by a dark brown staining in the cytoplasm of the neoplastic cells.

Abbreviations: HGIN, high-grade intraepithelial neoplasia; IGF2BP3, insulin-like growth factor-II mRNA-binding protein-3; IHC, immunohistochemistry.

Table 2 Immunohistochemical intensity data on IGF2BP3 expression

\begin{tabular}{llllllll}
\hline PT stage & $\mathbf{N}$ & \multicolumn{2}{l}{ Intensity } & & P-value \\
\cline { 3 - 6 } group & & Negative & Weak & Moderate & Strong & \\
\hline PT stage & 112 & 44 & 20 & 24 & 24 & 0.038 \\
PTIa-EP & 21 & 13 & 5 & 3 & 0 & \\
PTIa-LPM & 38 & 15 & 8 & 8 & 7 & \\
PTla-MM & 53 & 16 & 7 & 13 & 17 & \\
\hline
\end{tabular}

Notes: $\mathrm{PT}$ stage: pathological T stage; PT Ia-EP: MI, carcinoma in situ; PT Ia-LPM: M2, tumor-invaded lamina propria mucosa; PT la-MM: M3, tumor invaded the whole layer of mucosa. that ESCC derives from the basal layer of the squamous epithelium. ${ }^{20}$ Based on the results of Western blot and IHC analyses, it was found that IGF2BP3 was positive in approximately $60 \%$ of HGIN cases. The positive rate in HGIN was similar with that in advanced ESCC, ${ }^{9}$ which was $59.2 \%$. The positive rate of IGF2BP3 in squamous cell carcinoma of the skin, ${ }^{16}$ the head and neck, ${ }^{1,17}$ and the tongue ${ }^{18}$ were $57 \%, 61 \%$, and $77 \%$, respectively. According to the study by Kazeminezhad et al, ${ }^{15}$ the positive rate of IGF2BP3 was about 
Table 3 Correlation between IGF2BP3 expression and the patient demographics and endoscopic findings in HGIN

\begin{tabular}{|c|c|c|c|}
\hline \multirow[t]{2}{*}{ Characteristics } & \multicolumn{2}{|c|}{ IGF2BP3 expression } & \multirow[t]{2}{*}{$P$-value } \\
\hline & Positive $(n=68)$ & Negative $(n=44)$ & \\
\hline \multicolumn{3}{|l|}{ Gender } & 0.282 \\
\hline Male & 44 & 24 & \\
\hline Female & 24 & 20 & \\
\hline \multicolumn{3}{|l|}{ Age (years) } & 0.754 \\
\hline$\leq 50$ & 14 & 8 & \\
\hline$>50$ & 54 & 36 & \\
\hline \multicolumn{3}{|l|}{ Location } & 0.294 \\
\hline Upper & 16 & 16 & \\
\hline Middle & 19 & 12 & \\
\hline Lower & 33 & 16 & \\
\hline \multicolumn{3}{|l|}{ Length } & 0.731 \\
\hline$\leq 5 \mathrm{~cm}$ & 49 & 33 & \\
\hline$>5 \mathrm{~cm}$ & 19 & 11 & \\
\hline \multicolumn{3}{|c|}{ Circumferential extent } & 0.370 \\
\hline$<\mathrm{I} / 2$ & 15 & 15 & \\
\hline $1 / 2-2 / 3$ & 30 & 16 & \\
\hline$>2 / 3$ & 23 & 13 & \\
\hline \multicolumn{3}{|l|}{ pT stage } & 0.042 \\
\hline PTIa-EP & 8 & 13 & \\
\hline pTIa-LPM & 23 & 15 & \\
\hline pTIa-MM & 37 & 16 & \\
\hline
\end{tabular}

Notes: $\mathrm{PT}$ stage: pathological T stage; PTIa-EP: MI, carcinoma in situ; PTIa-LPM: M2, tumor-invaded lamina propria mucosa; PT Ia-MM: M3, tumor invaded the whole layer of mucosa.

Abbreviations: HGIN, high-grade intraepithelial neoplasia; IGF2BP3, insulin-like growth factor-II mRNA-binding protein-3.

91\% in advanced esophageal adenocarcinoma. In the study by Yantiss et al, ${ }^{21}$ IGF2BP3 was with a $97 \%$ positive rate in pancreatic adenocarcinoma. The positive rate of IGF2BP3 is also similar in esophageal adenocarcinoma and its precursor lesions. Using IHC analyses, Lu et a ${ }^{13}$ showed $94 \%$ positive expression of IGF2BP3 in the high-grade dysplasia/malignant glands. According to the available data, the positive rate of IGF2BP3 in adenocarcinomas seems higher than that in squamous cell malignancies. Compared with ESCC and HGIN, esophageal adenocarcinoma and its precursor lesion are with a higher positive rate of IGF2BP3. This difference may be due to different disease types. In malignant tumors, the overexpression of IGF2BP3 is associated with a worse prognosis or more advanced disease. ${ }^{22-24}$ In ESCC, IGF2BP3 overexpression may be associated with radioresistence. ${ }^{25}$ Furthermore, IGF2BP3 has been considered as a potential therapeutic target for ESCC. ${ }^{26}$ Although the functions of IGF2BP3 have been reported in ESCC, further studies will be performed to characterize the status of IGF2BP3 in HGIN.

In the cervical epithelium ${ }^{19}$ and the pancreatic ductal epithelium, ${ }^{21}$ IGF2BP3 expression increases with the degree of dysplasia. In this study, IGF2BP3 expression showed higher immunostaining in more advanced $\mathrm{pT}$ stage. Stronger staining intensity of IGF2BP3 was associated with the HGIN severity. The positive expression of IGF2BP3 was correlated with lesion depth, and this lesion depth was the only predictor of the positive expression of IGF2BP3. Our results were similar with previous reports. ${ }^{19,21}$ In our study, because all patients were free of lymph node lesions, the effect of pathological $\mathrm{N}$ stage and pathological stage (pStage) was not evaluated. Patients with IGF2BP3-positive ESCC experienced a poorer 5-year survival rate compared with those with IGF2BP3-negative tumors. ${ }^{9}$ However, in this study, data on survival were not analyzed since no patients died in 2-year follow-up periods.

\section{Conclusion}

The current study found that IHC of IGF2BP3 was reproducible and could be easily performed on HGIN tissues. The simplicity of the IHC assay will enable a preoperative diagnosis of biopsy tissues. The overexpression of IGF2BP3 may therefore be a supplement to the diagnosis of HGIN, especially in challenging cases. ${ }^{15}$ Overall, our results suggest a potential tissue marker for the diagnosis of HGIN and provide a complementary strategy to histopathologic examination. A large sample will be enrolled for further test validation.

Table 4 Univariate and multiple linear regression analysis for the correlations between the positive expression of IGF2BP3 and predictors

\begin{tabular}{|c|c|c|c|c|c|c|}
\hline \multirow[t]{2}{*}{ Predictors } & \multicolumn{3}{|c|}{ Univariate } & \multicolumn{3}{|c|}{ Multiple } \\
\hline & $\beta$ & Wald & $P$-value & $\beta$ & Wald & $P$-value \\
\hline Gender (male/female) & $0.4 \mathrm{II}$ & 0.967 & 0.326 & 0.429 & 0.991 & 0.319 \\
\hline Age $(\leq 50 />50$ years $)$ & -0.11 & 0.000 & 0.983 & 0.021 & 0.001 & 0.969 \\
\hline Location (upper/middle/lower) & -0.329 & 1.340 & 0.247 & -0.635 & 1.281 & 0.258 \\
\hline Length $(\leq 5 \mathrm{~cm} />5 \mathrm{~cm})$ & 0.133 & 0.073 & 0.787 & 0.091 & 0.034 & 0.854 \\
\hline Circumferential extent $(<1 / 2 / 1 / 2-2 / 3 />2 / 3)$ & -0.102 & 0.133 & 0.715 & -0.023 & 0.002 & 0.969 \\
\hline pT (pT Ia-EP/pTIa-LPM/pTIa-MM) & -0.647 & 5.508 & 0.019 & -1.343 & 5.257 & 0.022 \\
\hline
\end{tabular}

Notes: PT: pathological T; pT Ia-EP: MI, carcinoma in situ; PTIa-LPM: M2, tumor-invaded lamina propria mucosa; PTIa-MM: M3, tumor invaded the whole layer of mucosa. Abbreviation: IGF2BP3, insulin-like growth factor-II mRNA-binding protein-3. 


\section{Acknowledgments}

This work was funded by the Natural Science Foundation of Jiangsu Province, China (BK20151037). We thank all the patients enrolled in this study.

\section{Disclosure}

The authors report no conflicts of interest in this work.

\section{References}

1. Ferlay J, Soerjomataram I, Dikshit R, et al. Cancer incidence and mortality worldwide: source, methods and major patterns in GLOBOCAN 2012. Int J Cancer. 2015;136(5):E359-E386.

2. Kosugi S, Nishimaki T, Kanda T, et al. Clinical significance of serum carcinoembryonic antigen, carbohydrate antigen 19-9, and squamous cell carcinoma antigen levels in esophageal cancer patients. World $J$ Surg. 2004;28(7):680-685.

3. Bosman F, Carneiro F, Hruban RH, et al. WHO Classification of Tumors of the Digestive System. 4th ed. Lyon: IARC; 2010:18-24.

4. Okumura T, Shimada Y, Sakurai T, et al. Abnormal cell proliferation in the p75NTR-positive basal cell compartment of the esophageal epithelium during squamous carcinogenesis. Dis Esophagus. 2015;28(7): 634-643.

5. Teoh AY, Chiu PW, Yu Ngo DK, Wong SK, Lau JY, Ng EK. Outcomes of endoscopic submucosal dissection versus endoscopic mucosal resection in management of superficial squamous esophageal neoplasms outside Japan. J Clin Gastroenterol. 2010;44(9):e190-e194.

6. Wang GQ, Abnet CC, Shen Q, et al. Histological precursors of oesophageal squamous cell carcinoma: results from a 13 year prospective follow up study in a high risk population. Gut. 2005;54(2):187-192.

7. Müeller-Pillasch F, Lacher U, Wallrapp C, et al. Cloning of a gene highly overexpressed in cancer coding for a novel $\mathrm{KH}$-domain containing protein. Oncogene. 1997;14(22):2729-2733.

8. Mueller-Pillasch F, Pohl B, Wilda M, et al. Expression of the highly conserved RNA binding protein KOC in embryogenesis. Mech Dev. 1999;88(1):95-99.

9. Takata A, Takiguchi S, Okada K, et al. Expression of insulin-like growth factor-II mRNA-binding protein-3 as a marker for predicting clinical outcome in patients with esophageal squamous cell carcinoma. Oncol Lett. 2014;8(5):2027-2031.

10. Wang T, Fan L, Watanabe Y, et al. L523S, an RNA-binding protein as a potential therapeutic target for lung cancer. Br J Cancer. 2003;88(6): 887-894.

11. Okada K, Fujiwara Y, Nakamura Y, et al. Oncofetal protein, IMP3, a potential marker for prediction of postoperative peritoneal dissemination in gastric adenocarcinoma. J Surg Oncol. 2012;105(8):780-785.

12. Lederer M, Bley N, Schleifer C, et al. The role of the oncofetal IGF2 RNA-binding protein 3 (IGF2BP3) in cancer. Semin Cancer Biol. 2014; 29:3-12.
13. Lu D, Vohra P, Chu PG, Woda B, Rock KL, Jiang Z. An oncofetal protein IMP3: a new molecular marker for the detection of esophageal adenocarcinoma and high-grade dysplasia. Am J Surg Pathol. 2009;33(4): 521-525.

14. Dawsey SM, Fleischer DE, Wang GQ, et al. Mucosal iodine staining improves endoscopic visualization of squamous dysplasia and squamous cell carcinoma of the esophagus in Linxian, China. Cancer. 1998;83(2): 220-231.

15. Kazeminezhad B, Mirafsharieh SA, Dinyari K, et al. Usefulness of insulin-like growth factor II mRNA-binding protein 3 (IMP3) as a new marker for the diagnosis of esophageal adenocarcinoma in challenging cases. Turk J Gastroenterol. 2014;25:253-256.

16. Soddu S, Di Felice E, Cabras S, et al. IMP-3 expression in keratoacanthomas and squamous cell carcinomas of the skin: an immunohistochemical study. Eur J Histochem. 2013;57(1):e6.

17. Clauditz TS, Wang CJ, Gontarewicz A, et al. Expression of insulin-like growth factor II mRNA-binding protein 3 in squamous cell carcinomas of the head and neck. J Oral Pathol Med. 2013;42(2):125-132.

18. Li HG, Han JJ, Huang ZQ, Wang L, Chen WL, Shen XM. IMP3 is a novel biomarker to predict metastasis and prognosis of tongue squamous cell carcinoma. J Craniofac Surg. 2011;22(6):2022-2025.

19. Lu D, Yang X, Jiang NY, et al. IMP3, a new biomarker to predict progression of cervical intraepithelial neoplasia into invasive cancer. Am J Surg Pathol. 2011;35(11):1638-1645.

20. Imamura M. Superficial Esophageal Neoplasm: Pathology, Diagnosis, and Therapy. Japan: Springer; 2000.

21. Yantiss RK, Woda BA, Fanger GR, et al. KOC (K homology domain containing protein overexpressed in cancer): a novel molecular marker that distinguishes between benign and malignant lesions of the pancreas. Am J Surg Pathol. 2005;29(2):188-195.

22. Hsu KF, Shen MR, Huang YF, et al. Overexpression of the RNAbinding proteins Lin28B and IGF2BP3 (IMP3) is associated with chemoresistance and poor disease outcome in ovarian cancer. $\mathrm{Br} J$ Cancer. 2015;113(3):414-424.

23. Barton VN, Donson AM, Birks DK, et al. Insulin-like growth factor 2 mRNA binding protein 3 expression is an independent prognostic factor in pediatric pilocytic and pilomyxoid astrocytoma. J Neuropathol Exp Neurol. 2013;72(5):442-449.

24. Lochhead P, Imamura Y, Morikawa T, et al. Insulin-like growth factor 2 messenger RNA binding protein 3 (IGF2BP3) is a marker of unfavourable prognosis in colorectal cancer. Eur J Cancer. 2012;48(18): 3405-3413.

25. Yoshino K, Motoyama S, Koyota S. Identification of insulin-like growth factor $2 \mathrm{mRNA}$-binding protein 3 as a radioresistance factor in squamous esophageal cancer cells. Dis Esophagus. 2014;27(5):479-484.

26. Kono K, Iinuma H, Akutsu Y, et al. Multicenter, phase II clinical trial of cancer vaccination for advanced esophageal cancer with three peptides derived from novel cancer-testis antigens. J Transl Med. 2012;10:141.
OncoTargets and Therapy

\section{Publish your work in this journal}

OncoTargets and Therapy is an international, peer-reviewed, open access journal focusing on the pathological basis of all cancers, potential targets for therapy and treatment protocols employed to improve the management of cancer patients. The journal also focuses on the impact of management programs and new therapeutic agents and protocols on

\section{Dovepress}

patient perspectives such as quality of life, adherence and satisfaction. The manuscript management system is completely online and includes a very quick and fair peer-review system, which is all easy to use. Visit http://www.dovepress.com/testimonials.php to read real quotes from published authors. 